\title{
tic\&société
}

Vol. 14, $\mathbf{N}^{\circ}$ 1-2 | 1er semestre 2020 - 2ème semestre 2020

Mutations numériques de la musique : des

contradictions à analyser

\section{Revendiquer le salariat ou comment court-circuiter le cycle d'accumulation du capital des médias socionumériques}

EditorialPaid wages as a way to short circuit the accumulation of capital by

social media conglomerates

EditorialReivindicar el empleo asalariado, o cómo cortocircuitar el ciclo de acumulación de capital en las redes sociales

Samuel Lamoureux

\section{OpenEdition}

Journals

Édition électronique

URL : http://journals.openedition.org/ticetsociete/5338

DOI : 10.4000/ticetsociete.5338

Éditeur

Association ARTIC

Édition imprimée

Pagination : 343-373

Référence électronique

Samuel Lamoureux, «Revendiquer le salariat ou comment court-circuiter le cycle d'accumulation du capital des médias socionumériques », tic\&société [En ligne], Vol. 14, № 1-2 I 1er semestre 2020 2ème semestre 2020, mis en ligne le 11 novembre 2020, consulté le 26 février 2021. URL : http:// journals.openedition.org/ticetsociete/5338; DOI : https://doi.org/10.4000/ticetsociete.5338 
tic\&société - 14(1-2), 2020

\section{Revendiquer le salariat ou comment court-circuiter le cycle d'accumulation du capital des médias socionumériques}

\section{Samuel Lamoureux}

Samuel Lamoureux est étudiant au doctorat en communication à l'Université du Québec à Montréal. II se spécialise en études médiatiques et en critique de l'économie politique. Son mémoire de maîtrise (2019) portait sur une analyse critique des communications de la Banque du Canada ainsi que sur les médias traitant ces communications. Auxiliaire de recherche au Centre de recherche interuniversitaire sur la communication, l'information et la société (CRICIS), il a publié dans les revues Communiquer, Les Cahiers du Journalisme, Radiomorphoses, Québec Science et COMMposite.

lamoureux.samuel@courrier.uqam.ca 
Revendiquer le salariat ou comment court-circuiter le cycle d'accumulation du capital des médias sociaux numériques

\title{
Revendiquer le salariat ou comment court-circuiter le cycle d'accumulation du capital des médias sociaux numériques
}

Résumé : Cet article propose d'adopter une ancienne revendication pour enrayer un nouveau problème. Face à l'exploitation du travail des usagers des médias sociaux numériques, nous proposons que le meilleur moyen de contrer le cycle d'accumulation du capital des grandes corporations numériques est de revendiquer le salariat. Cette redistribution, qui pourrait prendre la forme d'un revenu contributif, viendrait directement ébranler les fondations du capitalisme financier.

Mots-clés : salariat, revenu contributif, médias socionumériques, exploitation, digital labor.

\section{Paid wages as a way to short circuit the accumulation of capital by social media conglomerates}

\begin{abstract}
This article proposes adopting an old proposal to solve a new problem. Given the exploitation of the work of digital social network users, we propose that the best way to counter the capital accumulation cycle of large digital corporations is to claim wage labor. This redistribution, which could take the form of a contributory income, would directly undermine the foundations of financial capitalism.
\end{abstract}

Keywords: wage claim, contributory income, social networks, exploitation, digital labor. 


\section{Reivindicar el empleo asalariado, o cómo cortocircuitar el ciclo de acumulación de capital en las redes sociales}

Resumen: Este texto propone la adopción de una antigua reivindicación para abordar un nuevo problema. Frente a la explotación del trabajo de los usuarios de las redes sociales digitales, establecemos que la mejor forma de contrarrestar el ciclo de acumulación de capital de las grandes corporaciones digitales es reivindicar el trabajo asalariado. Esta redistribución, que podría tomar la forma de un ingreso contributivs, socavaría directamente los cimientos del capitalismo financiero.

Palabras clave: trabajo asalariado, ingresos contributivos, redes sociales, explotación, trabajo digital. 



\section{Samuel LAMOUREUX}

\section{Introduction}

Les GAFAM ${ }^{1}$ sont maintenant les plus grandes corporations ${ }^{2}$ de notre capitalisme financier. La capitalisation boursière de Microsoft, par exemple, a dépassé le trillion de dollars en 2019 (Bekhtaoui, 2019). Cette concentration du pouvoir - car l'accumulation du capital est bien une quantification symbolique du pouvoir (Nitzan et Bichler, 2012) - menace les industries culturelles et le modèle d'affaires des médias traditionnels (Smyrnaios et Rebillard, 2019), mais surtout la démocratie moderne qui requiert les débats d'idées et la parole libérée au sein d'un monde commun (Freitag, 2011), plutôt que la communication dans des réseaux privés gérés par des algorithmes.

Pourtant, les penseurs critiques et les institutions politiques peinent à trouver des solutions face à l'expansion incontrôlable des grandes corporations numériques. Faudrait-il tout simplement taxer les GAFAM et redistribuer les montants (Belleflamme et Toulemonde, 2018), se retirer massivement de ces réseaux pour reconstruire nos communautés politiques (Ouellet, 2016), ou encore construire des réseaux authentiquement "sociaux" basés sur le principe des communs numériques (Lomazzi et Ménard, 2018; Scholz, 2017) ?

Inspirés par les premières luttes revendiquant le salariat étudiant, mais aussi le salariat des femmes ménagères (Robert, 2014), nous défendons dans ce texte le point de vue selon lequel la revendication du salariat (et surtout d'un revenu contributif) pour les utilisateurs des médias sociaux numériques serait le meilleur moyen d'ébranler le cycle d'accumulation du capital des grandes corporations numériques. Réclamer compensation pour du travail exploité non salarié est (historiquement) l'un des combats les plus efficaces pour faire

\footnotetext{
1 Google, Amazon, Facebook, Apple, Microsoft.

2 Le mot corporation est utilisé ici pour désigner la forme instituée de la grande entreprise dans le capitalisme financier. À la manière d'Éric Pineault (2008), le terme est " utilisé comme catégorie sociologique afin d'ancrer l'analyse de l'organisation capitaliste dans son développement typiquement anglo-américain et son institutionnalisation par la common law comme personne morale et puissance sociale légitime. [...] II ne s'agit pas donc pas d'un anglicisme, mais bien d'un concept ancré dans un procès socio-historique qui a été hégémonique au vingtième siècle » (p. 116).
} 
Revendiquer le salariat ou comment court-circuiter le cycle d'accumulation du capital des médias sociaux numériques

pression sur les fondations du capitalisme (Caffentzis et al., 2016). Ce constat était vrai sous le capitalisme monopoliste fordiste qui reposait sur l'exploitation du travail gratuit des femmes dans la sphère privée (Federici, 1975) ; il l'est encore aujourd'hui sous le capitalisme financier qui repose, lui, sur de multiples mécanismes d'accumulation différentielle qui ne proviennent bien souvent pas du travail salarié traditionnel (Nitzan et Bichler, 2012).

Notre étude s'inscrit à cet égard dans l'approche critique de l'économie politique de la communication qui met l'accent sur l'analyse de la constitution interne et de la dynamique du système économique (Mosco, 2008). La critique de l'économie politique vise à montrer les limites, les contradictions et les problèmes de l'économie capitaliste. Cette critique conteste la légitimité et la logique des approches théoriques qui conçoivent les variables capitalistes (comme la marchandise, la valeur, l'argent, le capital et la division du travail) comme universelles et non comme des variables historiques et contingentes (Fuchs, 2011).

Notre travail se situe dans une approche critique de l'économie politique de la communication parce qu'il vise à lever le voile sur le rôle des médias sociaux numériques dans la reproduction des rapports capitalistes de production, mais aussi parce qu'il cherche une porte de sortie à l'exploitation des usagers de ces médias sociaux numériques.

Ainsi, nous suggérerons un changement dans la conception de l'identité des usagers de ces médias, d'utilisateursbénévoles à travailleurs possiblement rémunérés. Pour ce faire, nous détaillerons premièrement notre cadre théorique, c'est-àdire la théorie de la valeur. Nous utiliserons successivement Marx au XIXème siècle, Smythe au XXème siècle et Fuchs au $X X \mid$ ème siècle. Nous expliquerons ensuite le changement d'identité que nous proposons (utilisateurs à travailleurs), nous détaillerons les notions de prosumer et de crowdsourcing, tout en introduisant les critiques à l'endroit du travail de Fuchs. Pour finir, nous discuterons de la question du salariat au XXIème siècle, des débats entre revenu universel et revenu contributif, et de l'enjeu de l'accélération de la marchandisation. 


\section{La théorie de la valeur}

\subsection{Une critique du fétichisme de la marchandise}

La théorie de la valeur est une critique du marxisme orthodoxe et de l'économie politique classique effectuée essentiellement par une relecture des écrits de Marx, surtout Le Capital. Cette approche critique les principales catégories de l'économie politique (travail, marchandise, valeur) en les considérant comme des fétiches ou des abstractions réelles qui sont à la base de l'objectivité sociale (Ouellet, 2015).

Le marxisme orthodoxe est l'idéologie sociale et politique qui a émergé des œuvres de Marx à partir de la fin du $\mathrm{XIX} \mathrm{ème}^{\mathrm{s}}$ siècle. Propagé surtout par les idéologues de la Deuxième internationale comme Lénine, le marxisme orthodoxe est dominé par l'idée d'un déterminisme technologique inhérent au progrès de l'histoire (Piccone, 1975). Ce progrès est expliqué par une tendance transhistorique au développement des forces productives. L'histoire est une téléologie, c'est-à-dire une succession de modes de production (esclavage, servage, salariat) qui doit mener à une phase supérieure du communisme. Chaque type de société est doté d'une base économique et d'une superstructure diffusant l'idéologie de la classe dominante (politique, religion, école, média).

La théorie de la valeur va à rebours du marxisme orthodoxe, car elle considère que des concepts comme le travail, la valeur, les marchandises et le capital sont des variables historiques propres au capitalisme :

Le produit du travail est, dans n'importe quel état social, valeur d'usage ou objet d'utilité ; mais il n'y a qu'une époque déterminée dans le développement historique de la société, qui transforme généralement le produit du travail en marchandise, c'est celle où le travail dépensé dans la production des objets utiles revêt le caractère d'une qualité inhérente à ces choses, de leur valeur (Marx, 1977, p. 61).

Dans ses écrits, Marx explique que le travail abstrait donne de la valeur aux marchandises. Dans le capitalisme, le sujet réifié est une chose mesurable en temps de travail. Autrement dit, la substance de la valeur est le travail, et la mesure de sa 
Revendiquer le salariat ou comment court-circuiter le cycle d'accumulation du capital des médias sociaux numériques

quantité est la durée du travail. "Ce n'est que dans le capitalisme que le travail, au lieu d'être "enchâssé" dans l'ensemble des relations sociales, comme c'était le cas dans les sociétés précapitalistes, devient lui-même un principe de médiation sociale » (Jappe, 2009, p. 101-102). La domination n'est donc plus seulement la domination de classe, mais le fait que la société est organisée autour du travail abstrait, le salariat étant au centre du lien social.

Pour Marx, l'élément fondamental du capitalisme est le fétichisme de la marchandise. La marchandise est créée par l'institutionnalisation de la norme temporelle qui associe le temps à l'argent. La valeur d'échange des choses supplante leurs valeurs d'usage. "C'est seulement dans leur échange que les produits du travail acquièrent comme valeurs une existence sociale identique et uniforme, distincte de leur existence matérielle et multiforme comme objets d'utilité » (Marx, 1977, p. 69). Par fétichisme de la marchandise, Marx entend étudier la perception que les acteurs ont des marchandises, comme des «êtres indépendants, doués de corps particuliers, en communication avec les hommes et entre eux » (Marx, 1977, p. 71).

Pour accumuler du capital, les capitalistes achètent de la force de travail et des moyens de production pour produire de nouvelles marchandises qui sont vendues pour acquérir du nouvel argent qui sera en partie réinvesti. Marx distingue deux sphères d'accumulation du capital : la sphère de la circulation et la sphère de la production (Fuchs, 2010).

Dans la sphère de la circulation, l'argent est transformé en marchandise et les capitalistes achètent la force de travail et les moyens de production. Dans la sphère de production, un nouveau bien est produit, augmenté par la valeur de la force de travail et des moyens de production. De retour dans la sphère de la circulation, la marchandise est vendue sur le marché à un prix supérieur à son coût de production, ce qui provoque une plus-value. Le capital monétaire initial M devient M'. Une partie de $M$ ' est réinvestie pour continuer à faire "tourner la roue ». La plus-value dans ce schéma est générée par le travail non payé (le surtravail) des ouvriers (Fuchs, 2010).

Toutefois, selon la théorie de la valeur, la production capitaliste de marchandises contient à la base une 
contradiction interne très importante: "On ne peut faire fructifier le capital, et donc l'accumuler, qu'en exploitant la force de travail. Mais le travailleur, pour engendrer un profit pour son employeur, doit être équipé des outils nécessaires, et aujourd'hui des technologies de pointe » (Jappe, 2011, p. 33). Pour être le plus performant possible, le capitaliste doit acquérir la meilleure technologie disponible, mais cette même technologie vient progressivement remplacer la force de travail qui est justement la source principale de profit. Le développement des technologies diminue les profits dans leur totalité. Moins une marchandise contient de travail, moins elle a de valeur. C'est ce que Marx a appelé la « baisse tendancielle du taux de profit $»$.

Le capitalisme a historiquement évité de s'écrouler en augmentant continuellement sa production de marchandises à des sphères nouvelles de la vie. "Pendant très longtemps, l'expansion interne et externe de la production des marchandises (vers d'autres régions du monde et à l'intérieur des sociétés capitalistes) a pu compenser la valeur amoindrie des marchandises particulières " (Martin et Ouellet, 2014, p. 57). Le développement de l'industrie culturelle et des médias de masse au XXème siècle est un bon exemple de l'extension du capitalisme vers la sphère de la culture et de la colonisation croissante de nos vies quotidiennes par ce système. II ne faut pas oublier que le capitalisme, à ses débuts au XIXème siècle (et bien avant si on observe le cas de l'Angleterre), était essentiellement agraire (Wood, 2002). Le développement de la société de consommation au XXème siècle était un moyen d'étendre la sphère de la valeur au temps de loisir des travailleurs.

\subsection{Smythe et l'audience-marchandise}

La pensée de Dallas Smythe et surtout son article "Communications: Blindspot of western Marxism ", publié en 1977, est l'un des meilleurs moyens de faire le pont entre la théorie de la valeur et les enjeux reliés à l'économie politique de la communication. Marx décrivait un capitalisme industriel rythmé par l'opposition entre le bourgeois et le prolétaire. Le $X X^{\text {ème }}$ siècle voit plutôt l'apparition, d'abord aux États-Unis, d'un capitalisme monopoliste mené par la surproduction et la surconsommation. La classe de travailleurs devient donc une 
Revendiquer le salariat ou comment court-circuiter le cycle d'accumulation du capital des médias sociaux numériques

classe de consommateurs (Vioulac, 2018).

Smythe critique en ce sens les auteurs marxistes traditionnels qui analysent les messages des médias dans le but d'y trouver une idéologie quelconque. Celui-ci affirme plutôt qu'il faudrait comprendre le rôle des médias dans la reproduction des rapports capitalistes de production. La thèse de Smythe est la suivante : la fonction des médias de masse est de produire un public (audience), qui prend la forme d'une marchandise qui a de la valeur, pour ensuite la vendre aux annonceurs.

Le travailleur moyen du $X X^{\text {ème }}$ siècle travaille beaucoup moins d'heures par semaine qu'un travailleur moyen du XIXème siècle. Cette situation est le résultat de nombreuses luttes syndicales pour la diminution du temps de travail. Toutefois, dit Smythe, le capitalisme s'est ajusté à cette réalité en colonisant le temps de loisir des travailleurs, c'est-à-dire en le transformant en un temps de consommation. En effet, les travailleurs, observait Smythe en 1977, passent plus de 40 heures par semaine comme public d'un grand média. Ils sont donc exposés en permanence à de la publicité et soumis à une énorme pression par tous les biens de consommation du capitalisme monopolistique. Par grand média, l'auteur entend la radio, la télévision, les journaux et les magazines.

En formant l'auditoire des médias de masse, les travailleurs apprennent à dépenser leur salaire sur des biens de consommation et, par conséquent, à simultanément reproduire leur propre force de travail (Smythe, 1977). Tout le temps est donc du temps de travail, excepté le temps de sommeil ${ }^{3}$ :

\footnotetext{
${ }^{3}$ Bien sûr, la théorisation de Smythe a donné lieu à de nombreux débats (Manzerolle et McGuigan, 2014). Pour certains, ce ne serait pas les auditeurs qui travailleraient, mais bien plus les statisticiens et les analystes qui recueillent leurs informations (Bolin, 2010). De notre côté, nous suivons les écrits de Huws (2014) sur le digital labor, qui argumente toute l'importance du travail de consommation, un travail non payé qui est de plus en plus important dans le capitalisme postfordiste et qui comprend le travail des auditeurs introduit par Smythe. Le travail de consommation est essentiellement un travail qui facilite la distribution de la marchandise vers son public (Dujarier, 2008; Huws, 2014). Cela peut être le décodage des messages publicitaires de la télévision au $X X^{\text {ème }}$ siècle tout comme le fait de réaliser une vidéo d'influenceur pour présenter des marques de cosmétique aujourd'hui. Toutefois, comme dit Casilli (2019), le travail de consommation aujourd'hui n'est plus seulement de regarder, mais aussi d'être regardé par les mécanismes de surveillance des plateformes numériques.
} 
Les institutions des médias de masse dans le capitalisme monopoliste ont développé les équipements, les travailleurs et l'organisation nécessaires pour produire des audiences aux fins du système entre 1875 et 1950. Le but premier des médias de masse est de produire une audience qui travaille à apprendre la consommation de biens civils et qui soutient le système de la demande militaire ${ }^{4}$. (Smythe, 1977, p. 20.)

Contrairement aux analyses marxistes traditionnelles, le rôle des médias de masse n'est pas de produire une idéologie contenue dans une superstructure. Dans la théorie de Smythe, les médias de masse sont à la fois impliqués dans la superstructure et dans la base, où ceux-ci sont indispensables pour la stimulation (et la satisfaction) de la demande pour les produits de consommation. Si l'auteur affirme être le premier à formuler cette théorie, il n'a pas tout à fait raison. L'École de Francfort avait abordé la problématique de la reproduction de la force de travail dans ses écrits sur l'industrie culturelle : " Dans le capitalisme avancé, l'amusement est le prolongement du travail. II est recherché par celui qui veut échapper au processus de travail automatisé pour être de nouveau en mesure de l'affronter " (Horkheimer et Adorno, 1983, p. 203). Toutefois, l'apport de l'École de Francfort concerne plus l'aliénation et la réification que l'exploitation.

\subsection{Le capitalisme de Google}

La montée d'un capitalisme financier au XXIème siècle mené par les grandes corporations numériques dont le rythme d'accumulation repose sur leur capacité à anticiper leur profit dans le futur (Nitzan et Bichler, 2012) change encore la façon de concevoir l'exploitation. Depuis le début des années 2000, de nombreux chercheurs ont à cet égard analysé la création de la valeur par les médias sociaux numériques (MSN) dans le capitalisme financier (certains vont parler de capitalisme « de plateforme »(Srnicek, 2018)). Tiziana Terranova a été l'une

4 Traduction libre de: "the mass media institutions in monopoly capitalism developed the equipment, workers and organization to produce audiences for the purposes of the system between about 1875 and 1950. The prime purpose of the mass media complex is to produce people in audiences who work at learning the theory and practice of consumership for civilian goods and who support the military demand management system. » 
Revendiquer le salariat ou comment court-circuiter le cycle d'accumulation du capital des médias sociaux numériques

des premières à écrire sur le phénomène du «Free labor ». En effet, selon Terranova (2000), qui adopte la perspective marxiste de la valeur-travail, " la valeur économique des $\mathrm{MsN}$ proviendrait du surtravail non-rémunéré [sic] des usagers " (Ouellet et al., 2015, p. 603). Toutefois, Terranova s'appuie beaucoup sur la théorie des autonomistes italiens comme Negri et Lazzarato (travail immatériel) et, en ce sens, son apport n'est pas très intéressant pour notre travail.

Nous allons plutôt utiliser les travaux de Christian Fuchs, surtout « Labor in Informational Capitalism and on the Internet " (2012) et « Google capitalism » (2010). Fuchs emploie lui aussi parfois des concepts autonomistes (multitude), mais celui-ci se rapproche de nos objectifs en réactualisant la pensée de Dallas Smythe.

En 1977, Smythe décrivait une époque dominée par la publicité télévisuelle. Aujourd'hui, son analyse peut apparaître quelque peu désuète. "La théorie de Smythe sur l'audiencemarchandise a été développée à une époque dominée par la publicité télévisée où les chaînes étaient peu nombreuses et où la télécommande n'était pas encore un appareil largement

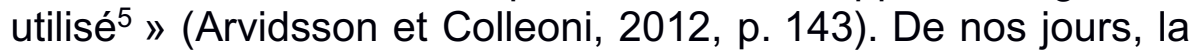
publicité a largement migré vers les médias sociaux numériques. Fuchs considère cependant que le modèle original de Smythe peut très bien être transposé vers ces médias.

En effet, pour Christian Fuchs (2010), les usagers de médias socionumériques constituent aussi un public-marchandise vendu aux annonceurs. La différence entre les deux publics est que celui des médias socionumériques est beaucoup plus actif que le public de la télévision. Bien sûr, le public de la télévision a toujours été impliqué dans du travail de consommation (Huws, 2014), mais il n'a jamais été jusqu'à créer son propre contenu de manière systématique comme le public des médias socionumériques, qui est activement impliqué dans la création/production/révision de contenus (Cardon et Casilli, 2015). La capacité de surveillance des corporations numériques est aussi beaucoup plus développée que celle des médias de masse du XXème siècle (Andrejevic, 2013). Ainsi,

\footnotetext{
${ }^{5}$ Traduction libre de: "Smythe's theory of the audience commodity was developed in an era dominated by television advertising where channels were few and the remote control was not yet a widely used device" ".
} 
pour Fuchs, la principale nouveauté du modèle économique des MSN résiderait donc dans le fait que «les informations personnelles récoltées au moyen des techniques de surveillance des consommateurs de plus en plus sophistiquées permettraient aux entreprises de diffuser des publicités de plus en plus ciblées en fonction des intérêts des usagers " (Ouellet et al., 2015, p. 603).

L'analyse de Fuchs sur le travail des utilisateurs étant explicitée au prochain point, nous allons nous concentrer ici sur sa description du cycle d'accumulation du capital des médias socionumériques présentée dans l'article « Google capitalism ».

Fuchs écrit que Google est l'une des compagnies les plus profitables au monde - Alphabet a récolté 32,3 milliards de revenus dans le dernier quart de 2017 (Statt, 2018) -, mais la question qu'il faudrait se poser est de savoir comment la compagnie engendre ses profits. Contrairement au capitalisme monopoliste de type informationnel ${ }^{6}$, qui vise à produire et à commercialiser des biens à base informationnels (molécules, semences, etc.) protégés par des droits de propriété intellectuelle qui permettent de vendre chaque copie à un prix très supérieur à son coût de production, le capitalisme de Google est plutôt caractérisé par l'exploitation des données personnelles et l'intermédiation algorithmique (Broca, 2018). Comme l'écrit Srnicek (2018), Google, tout comme Facebook, représente très bien le modèle de la plateforme publicitaire monopoliste qui vise à extraire l'information des usagers, à en faire l'analyse et à la vendre aux annonceurs, aux agences de crédit, aux assureurs ou aux services de police.

Voici donc comment se déploie le cycle d'accumulation du capital de Google selon Fuchs. Premièrement, Google investit de l'argent pour acheter du capital: des technologies et des travailleurs. Les employés de Google produisent des services comme Gmail, YouTube, etc. Les services de Google ne sont pas des marchandises, ils ne sont pas vendus aux utilisateurs. Ils sont fournis gratuitement. Cette gratuité et le grand nombre de services attirent le public. Le Google Search, qui est le service principal de Google, est alimenté par du travail non payé du public (comme tous les autres services de Google).

6 Pour une introduction au concept de capitalisme informationnel, voir Castells (2001). 
Revendiquer le salariat ou comment court-circuiter le cycle d'accumulation du capital des médias sociaux numériques

Google collecte les données de ses utilisateurs. Cette nouvelle marchandise est vendue aux annonceurs. Google a de l'argent nouveau (M'), et le cycle recommence (Fuchs, 2012).

Les utilisateurs de Google constituent un objet double de marchandisation : ils sont vendus avec leurs données comme marchandises et, par cette marchandisation, leur conscience est exposée de manière permanente à la publicité. "Most online time is advertising time " (Fuchs, 2012, p. 44). Puisque Google vend les données des utilisateurs, il doit aussi les emmagasiner. Google doit par conséquent s'engager dans la surveillance économique des données et des activités de l'utilisateur.

La règle d'or du capitalisme sur Internet est la suivante : la plateforme qui possède le plus d'utilisateurs engendre les plus hauts prix publicitaires. La marchandise n'est pas les services de Google, mais les utilisateurs et leurs données. Si la marchandise était les services, il faudrait payer pour utiliser Gmail ou YouTube, mais ces plateformes sont gratuites. L'argent est donc monétisé ailleurs. Examinons à présent le travail des utilisateurs.

\section{D'utilisateurs à travailleurs}

\subsection{Digital labor, crowdsourcing et prosommation}

Un des aspects très importants du cycle d'accumulation du capital de Google décrit par Fuchs est le travail des utilisateurs. Cette variable n'était pas présente avant Internet: dans le modèle de Smythe, le public est relativement passif face aux médias de masse. Son attention doit être constamment sollicitée pour être réceptive et pour décoder le grand nombre de publicités (Smythe, 1977). Sur les médias socionumériques, les utilisateurs sont plutôt impliqués dans la production de contenu et donc effectuent un travail : un digital labor, défini comme les activités numériques quotidiennes des usagers des plateformes sociales, des objets connectés ou des applications mobiles (Cardon et Casilli, 2015, p. 13). Sur Google, le travail peut prendre les différentes formes suivantes: chercher un mot-clé, envoyer un Gmail, chercher ou téléverser une vidéo sur YouTube, chercher un livre sur Google Print, chercher un endroit sur Google Maps, créer un document sur Google Docs, 
traduire une phrase avec Google translate, etc. (Fuchs, 2012.) Et c'est le lien de tous ces travaux et leur mise en relation avec ceux des autres qui produit les métadonnées à extraire qui ont de la valeur (Casilli, 2019).

Le digital labor est donc une nébuleuse qui inclut autant du travail créatif, du travail de consommation et ce que Jarrett (2015) nomme du "travail domestique numérique », c'est-àdire du travail affectif, considéré comme improductif et, surtout, effectué par les femmes. Certaines formes de digital labor sont déjà rémunérées, quoique très mal et surtout à la pièce, mais la majorité de ce travail n'est pas rémunéré (Casilli, 2019). Tout digital labor entre également dans une logique de production de valeur, d'encadrement de la participation et de disciplinarisation et de quantification de la force de travail (Andrejevic, 2013).

Une des caractéristiques principales du digital labor est de brouiller la frontière entre production et consommation. En effet, puisque les utilisateurs de Google ne sont pas seulement des consommateurs, mais aussi des producteurs, Fuchs affirme qu'ils deviennent des prosumers. Le terme prosumer a été élaboré par Alvin Toffler au début des années 1980. Chez Toffler (1980), ce concept signifie le déclin de la frontière qui sépare le producteur du consommateur. Pour les entreprises, l'utilisation de prosumer est un gain important de productivité, puisque le consommateur effectue des tâches qui incombaient auparavant à des employés salariés (Goyette-Côté, 2013). Ritzer (1983) va quant à lui parler de " McDonalization » de la société pour décrire cette nouvelle implication des consommateurs. Le rôle des prosumers est par conséquent de maximiser la plus-value dans une logique d'accumulation du capital. Les marchandises sont vendues au même prix, mais elles contiennent moins de capital variable, ce qui permet d'augmenter la marge de profit. Pour Fuchs, le savoir et la richesse ne sont pas seulement produits par les entreprises dans le capitalisme numérique, mais bien par une multitude de prosumers. Le problème est que cette production est appropriée par le capital.

Pour Goyette-Côté (2013), l'activité des usagers des plateformes participatives, loin d'être émancipatrice, doit également être perçue comme une forme de crowdsourcing, " dans la mesure où ce sont les entreprises capitalistes qui possèdent les moyens de production, les droits sur les produits 
Revendiquer le salariat ou comment court-circuiter le cycle d'accumulation du capital des médias sociaux numériques

générés et les profits engendrés par l'activité des internautes » (p. 151).

Le concept de crowdsourcing 2.0 a d'abord été défini par Jeff Howe dans la revue Wired en 2006. Ce concept est essentiellement l'idée d'externaliser du travail vers le public. Pour Howe (2006), « [l]e nouveau bassin de main-d'œuvre bon marché consiste en des personnes ordinaires utilisant leurs [ressources] pour créer du contenu, résoudre des problèmes et même effectuer de la recherche et du développement ${ }^{7}$ " (p. 1). Selon l'auteur, de plus en plus d'entreprises feraient appel à des individus du grand public pour effectuer des tâches auparavant complétés par des employés.

Ces tâches peuvent, par exemple, être celles d'un journaliste qui veut trouver un sujet, ou encore celle d'une compagnie qui se cherche un nouveau logo, un nouveau slogan, etc. Le terme crowdsourcing « tire son origine des mots anglais crowd (foule) et outsourcing (délocalisation (offshore)). II s'agit donc de sortir des frontières nationales (offshoring) ou de l'entreprise (outsourcing) en faisant appel, soit à des entreprises tierces, soit à la population (crowdsourcing) " (Goyette-Côté, 2013, p. 154).

L'idée derrière l'utilisation du crowdsourcing est que le web se rapproche d'une intelligence collective, d'un lieu fédérateur de connaissances où l'on peut piger du savoir commun. « Nous voyons donc que le crowdsourcing permet d'incorporer les prosumers dans le cycle de production, de les intégrer dans le processus de création de la valeur et, en particulier, qu'ils sont intégrés dans les stratégies des entreprises " (Goyette-Côté, 2013, p.155).

Un des résultats les plus néfastes de ce phénomène est de brouiller les cartes entre temps de loisir et temps de travail. Avec Smythe, le temps de loisir était transformé en un temps de consommation. Maintenant, avec Fuchs, le temps de loisir est transformé en un temps de travail. Ainsi, tout le temps est du temps de travail actif, excepté le temps de sommeil (Cardon et Casilli, 2015). Le travailleur autonome illustre bien la difficulté de séparer le temps de travail du temps de loisir. Celui-ci peut gérer ses heures de travail, mais plusieurs de ses actions non

7 Traduction libre de : "The new pool of cheap labour is everyday people using their spare [ressources] to create content, solve problems, even do corporate R\&D ». 
rémunérées sont nécessaires à l'exercice de son travail (par exemple, l'acquisition de connaissances, les formations, l'achat de matériel, etc.) (Goyette-Côté, 2013, p. 156). La figure du travailleur autonome est particulièrement importante dans notre époque hyperindividualiste qui favorise le développement des « autoentrepreneurs » (Lazzarato, 2011, p. 75).

Le temps passé sur les médias socionumériques, considéré comme un temps de loisir, pose problème dans la mesure où les utilisateurs de ces plateformes ne sont pas seulement des consommateurs, mais aussi des producteurs et donc des travailleurs :

Par conséquent, les victimes d'exploitation de la plusvalue dans des cas tels que Google, YouTube, MySpace ou Facebook ne sont pas simplement les personnes employées par ces sociétés pour la programmation, la mise à jour et la maintenance du logiciel et du matériel, mais également les utilisateurs engagés dans la production de contenus originaux ${ }^{8}$ (Fuchs, 2010, p. 191).

Pour Casilli, un autre problème posé par le digital labor est qu'il s'inscrit dans une logique de déqualification. La digitalisation des tâches humaines est pour lui une standardisation et une externalisation des tâches (Casilli, 2019). Le travail effectué sur les plateformes numériques capitalistes constitue rarement des tâches complexes. Au contraire, celui-ci est bien souvent divisé en micro-tâches ou en travail à la pièce qui ne s'inscrit pas dans une perspective d'émancipation (Casilli, 2019). II existe donc une tension constitutive à l'intérieur du digital labor qui est celle du contrôle du procès de travail et de sa déqualification ou requalification constante, et à l'inverse l'effort des travailleurs pour reconquérir un travail qui fait du sens et qui n'est pas coincé dans une passivité d'usager.

Face à cette problématique, face à cette nouvelle forme d'exploitation, face au déclin du temps de loisir, nous en arrivons à la proposition de notre travail : la redistribution. Si les plateformes participatives font travailler le public (et

8 Traduction libre de: "Therefore, the victims of exploitation of surplus value in cases like Google, YouTube, MySpace, or Facebook are not merely those employed by these corporations for programming, updating, and maintaining the soft and hardware, but also the users and the produsers engaged in the production of usergenerated content ». 
Revendiquer le salariat ou comment court-circuiter le cycle d'accumulation du capital des médias sociaux numériques

transforment leur temps de loisir en temps de travail), nous soutenons que le meilleur moyen de mettre le doigt sur le problème est de demander compensation pour le travail non payé. $\mathrm{Si}$ les usagers des médias socionumériques considéraient leur temps passé en ligne comme un temps de travail, nous pourrions assister à un revirement de situation complet quant à la colonisation des médias socionumériques par les GAFAM (Broca, 2018).

\subsection{Financiarisation de l'économie et rente informationnelle}

Bien que nous utilisons les analyses de Fuchs parues dans son article "Labor in Informational Capitalism and on the Internet», nous devons souligner quelques éléments problématiques de son cadre théorique.

Premièrement, l'emploi du concept postmarxiste de " multitude » par Fuchs n'est pas le meilleur moyen d'aborder la question de l'exploitation dans le capitalisme numérique. Chez l'auteur, la multitude regroupe autant les travailleurs industriels traditionnels, les travailleurs du savoir, les femmes de ménage, les chômeurs, les migrants, les retraités et les étudiants (Fuchs, 2010). Presque toute la population est représentée par ce concept, ce qui fait dire à Arvidsson et Colleoni (2012) que celui-ci est beaucoup trop vague pour être utilisée en tant qu'alternative au concept de classe sociale (Arvidsson et Colleoni, 2012 ; Huws, 2014).

Néanmoins, la critique principale des deux auteurs vise surtout la création de la valeur des médias socionumériques. Chez Fuchs, les profits de Google sont produits par le travail non payé des utilisateurs. II s'agit somme toute d'une interprétation très orthodoxe de la théorie de la valeur-travailabstrait où le nombre exact d'heures de travail en ligne déterminerait directement la valeur. D'autres auteurs ont apporté avec raison certaines corrections à cette thèse. La critique la plus importante est que Fuchs ne prend pas en considération la catégorie de la rente (Nixon, 2014). Premièrement, Arvidsson et Colleoni, qui ont font partie des premiers à avoir lancé le débat, affirment que la création de la valeur sur ces plateformes n'est pas assez liée à une quantité de temps productifs. Pour eux, la création de la valeur ne 


\section{Samuel LAMOUREUX}

devrait pas seulement prendre en compte la vente de marchandises, mais aussi le rôle central de la finance et des marchés financiers. Dans les médias socionumériques,

[l]a réalisation de la valeur ne se produit pas principalement par l'intermédiaire d'un échange direct de marchandises de base lorsque les prix du marché correspondent au temps de travail nécessaire, mais à travers des formes d'échange de marchandises médiatisées où des facteurs tels que la réputation, la marque et les conventions appuyés par des instruments affectifs interviennent pour fausser, obscurcir et médiatiser cette relation ${ }^{9}$ (Arvidsson et Colleoni, 2012, p. 142).

Toutefois, Arvidsson et Colleoni, en plus d'entretenir certaines confusions sur la notion de valeur (Goyette-Côté et Langlois, 2013), utilisent un concept, la valeur affective, très peu théorisé. L'accumulation du capital dans le capitalisme financier repose plutôt sur le pouvoir de capitalisation des entreprises, qui est la valeur actualisée d'un flux de revenus futur (Nitzan et Bichler, 2012).

Une autre critique plus détaillée est celle d'Ursula Huws dans ses articles sur le cybertariat. Celle-ci explique que le capital accumulé par les GAFAM ne provient pas que de la vente de données personnelles dans un cycle de production, mais aussi de la catégorie de la rente. Elle compare le modèle d'affaires de Google, par exemple, aux commerces qui louent des panneaux publicitaires longeant les autoroutes. Ces commerces n'accumulent pas du capital en vendant des marchandises, mais bien en profitant de leur position stratégique. Les GAFAM font la même chose: ils créent une autoroute virtuelle qui permet aux annonceurs de rejoindre leur public. Le capital provient donc aussi de stratégies monopolistiques comme le contrôle de l'accès au marché, des processus de fusions et d'acquisitions et l'emprise sur la propriété intellectuelle grâce à l'introduction de nombreux brevets (Smyrnaios, 2017). C'est d'ailleurs ce pour quoi Durand (2018) écrit que le numérique est un capitalisme intellectuel monopoliste. Mobiliser la catégorie

9 Traduction libre de: "Value realization does not primarily occur through direct commodity exchange where market prices correspond to necessary labor time, but through mediated forms of commodity exchange where factors like reputation, brand, and value conventions that are supported by public affective instruments intervene to distort, obscure, and mediate this relationship ». 
Revendiquer le salariat ou comment court-circuiter le cycle d'accumulation du capital des médias sociaux numériques

de la catégorie de la rente dans les analyses critiques n'infirme toutefois pas l'importance du travail et de la redistribution: le capital des corporations numériques provient bel et bien de l'activité de leurs usagers.

De manière plus générale, d'autres auteurs se sont aussi questionnés sur le fait que d'être actif en ligne constitue vraiment la réalisation d'un travail (Hesmondhalgh, 2010; Scholz, 2013). Certaines plateformes ne tireraient pas profit du travail de leurs utilisateurs, mais bien de leur présence en ligne ou de leur connexion permanente (Andrejevic, 2013). L'Internet des objets permettrait de subsumer en tout temps nos interactions, et pas seulement lorsque nous travaillons (Saleh, 2017). Pour Cardon (Cardon et Cassili, 2015), les critiques du digital labor jugent de haut les internautes en les condamnant d'avance à la posture de l'exploité sans prendre en compte leur expérience.

Loin de constituer une réfutation de l'argument du travail, nous croyons que ces remarques trahissent tout d'abord une vision simpliste du travail (et de l'exploitation) qui est beaucoup plus complexe qu'une simple activité productive encadrée par des capitalistes dans une relation salariale. Huws, par exemple, a longuement développé sur le travail «improductif mais nécessaire ", comme le travail domestique qui reproduit les relations sociales à l'extérieur de l'usine ou encore le travail de consommation qui effectue une partie des tâches réservées à la sphère de la distribution des marchandises (De Kosnik, 2013 ; Huws, 2014). Ces deux types de travail sont invisibles, mais fondamentaux dans la relation d'exploitation entre le capital et le travail, et ils représentent une grande partie du travail effectué en ligne (Jarrett, 2015). Ensuite, la mutation des activités en ligne ne signifie pas pour nous un déclin du travail, mais plutôt les efforts des grandes entreprises numériques pour constamment déqualifier, standardiser, externaliser et parcelliser le travail, un processus capitaliste constant $^{10}$ (Braverman, 1976 ; Casilli, 2019). Cela légitime encore plus le fait de vouloir demander rétribution pour stimuler la lutte des

\footnotetext{
10 Comme le dit également Casilli (2019), les plateformes numériques nous mettent au travail, mais aussi hors travail, c'est-à-dire en dehors des « modalités classiques de la relation d'emploi » (p. 26). De là découle toute la difficulté à considérer le digital labor comme du travail, ce travail ne fournissant pas des emplois, mais bien des tâches parcellisées.
} 
usagers, qui se retrouvent bien souvent atomisés devant leur écran. Comme l'écrit Casilli (dans Cardon et Casilli, 2015) : « la notion de digital labor refuse de faire l'impasse sur les phénomènes de captation de la valeur par le capitalisme des plates-formes numériques » (p. 15).

\section{Sur le salariat}

\subsection{Revenu universel et revenu contributif}

À la fin de son article «Labor in Informational Capitalism and on the Internet » (2010), Fuchs suggère que l'instauration d'un revenu universel garanti pourrait être un moyen de combattre l'exploitation du travail non payé des utilisateurs du web 2.0. Dans son article "Google capitalism » (2012), il propose plutôt la création d'un Google public soutenu par des universités. Sébastien Broca (2018) suggère quant à lui trois hypothèses de réponse possibles, dont deux qui regroupent les idées de Fuchs: le revenu contributif, le revenu d'existence, ou encore des nouveaux types d'entreprises (plateformes coopératives).

Nous examinerons ici les deux premières propositions qui nous semblent plus réalistes, étant donné que le revenu universel est une proposition politique concrète envisagée par plusieurs États occidentaux dans les dernières années (Salvet, 2018). Pour l'instant, la création de plateformes coopératives populaires ou la nationalisation de plateforme existante ne semblent pas être des solutions qui ont beaucoup d'élan malgré l'effort soutenu de certains chercheurs (Sandoval, 2020; Scholz, 2017 $)^{11}$. De plus, les plateformes coopératives, comme Resonate.is pour la musique, sont parfois de vains efforts devant la puissance monopolistique des GAFAM renforcée par un effet de réseau constant (le fait qu'un grand nombre de public attire toujours plus de public (Srnicek, 2018)).

Fuchs ne détaille pas sa proposition de revenu universel, mais, essentiellement, l'idée d'un revenu minimum garanti consiste à instaurer un revenu versé sans condition par une communauté politique à tous ses membres, sur une base

11 II y a des solutions coopératives, mais celles-ci ne sont pas grand public et pourraient surtout se concrétiser dans des secteurs de niche. Voir : https://degooglisons-internet.org/ ou encore http://ioo.coop/democratizingdocuments/. 
Revendiquer le salariat ou comment court-circuiter le cycle d'accumulation du capital des médias sociaux numériques

individuelle. Le problème de cette proposition est qu'elle est d'origine néolibérale (bien qu'aussi soutenu par des politiciens de gauche aujourd'hui). En effet, Milton Friedman parlait déjà, dans les années 1960, de l'idée d'instaurer un impôt négatif. « Pour Friedman, l'impôt négatif entraînerait évidemment la fin des services publics, de la sécurité sociale et de toute forme de socialisation des revenus à des fins collectives " (Zamora, 2016, p. 23).

Le revenu universel est une proposition dangereuse puisque celle-ci pourrait très bien signifier le démantèlement des services de l'État. II s'agit d'une substitution de la lutte contre les inégalités par une lutte contre la pauvreté. « Fondamentalement, prôner l'instauration d'une allocation universelle, c'est abandonner la lutte contre les inégalités » (Zamora, 2016, p. 29). En ce sens, cette proposition pourrait très bien avoir un effet pervers.

Puisque notre objectif est de mettre le doigt sur une nouvelle réalité d'exploitation, les réflexions de Bernard Stiegler sur le revenu contributif sont beaucoup plus intéressantes. Pour Stiegler, le revenu universel et le revenu contributif répondent à des enjeux différents, mais complémentaires. L'un ne doit pas remplacer l'autre, il s'ajoute à celui-ci. Le revenu universel est un minimum vital, mais il ne crée aucune dynamique positive et est donc insuffisant. Le revenu contributif, quant à lui, « est un revenu conditionnel, qu'on ne peut renouveler [...] qu'à la condition de "recharger" ses droits grâce à l'acquisition mais aussi à la transmission de savoirs, savoir-faire et savoir-être " (Stiegler et Kyrou, 2016, p. 54). Le but d'un revenu contributif est d'en arriver à une société des savoirs basée sur la contribution et le partage de connaissances. Si un travailleur possède des connaissances particulières en informatique, en art plastique, en mathématique, etc., il est invité à partager cette connaissance dans un processus d'éducation populaire.

Dans le cas qui nous concerne, l'application d'un revenu contributif pour les médias socionumériques peut sembler dérisoire. II serait absurde, en effet, de payer quelqu'un pour simplement écrire des statuts d'humeur ou "liker » des publications. Mais le travail sur ces réseaux peut aller beaucoup plus loin que cela.

Broca (2018) rappelle que les activités des grandes 
compagnies numériques comme Facebook et Google reposent en bonne partie sur les efforts de logiciel libre comme Linux. Ces logiciels ont été développés au départ par des bénévoles, pour ensuite être récupérés par les GAFAM. Un revenu contributif pourrait très bien être appliqué aux travailleurs développant des logiciels libres, aux contributeurs de l'encyclopédie en ligne Wikipédia, mais aussi, plus simplement, à des utilisateurs ordinaires de Google et de Facebook qui contribueraient à l'éducation populaire en produisant, par exemple, des capsules vidéos ou des modes d'emploi quelconque. L'enjeu, ici, est de favoriser le développement d'une véritable économie des communs dans laquelle les ressources comme Linux ne servent pas simplement d'entrants gratuits pour les entreprises capitalistes (Broca, 2018). II faut plus qu'un régime juridique comme le Copyleft, qui favorise le partage des ressources, il faut une reconnaissance de la contribution aux communs et aux médias socionumériques comme un travail et non comme un hobby.

II faut toutefois souligner que les travaux de Stiegler s'insèrent dans une réflexion plus globale sur la société du futur dans laquelle celui-ci prédit la mort de l'emploi. Stiegler imagine la venue d'une société entropique créée par l'ubérisation de la société. Ses propositions sont un moyen de combattre ce futur en proposant une économie "néguentropique " (Stiegler et Kyrou, 2016, p. 51). Nous ne suivons ici pas nécessairement Stiegler dans sa vision pessimiste de l'avenir, mais nous reconnaissons que ses réflexions sur le revenu universel et le revenu contributif sont essentielles pour combattre l'exploitation dans le capitalisme numérique.

\subsection{Un moyen et non une fin}

Nous discuterons à présent des effets potentiellement néfastes que pourrait produire l'introduction d'une nouvelle forme de salariat dans la société. Considérée seulement comme une fin, la rémunération du travail sur les médias socionumériques pourrait provoquer une accélération de la marchandisation de la société (Hesmondhalgh, 2010). Cardon parle même d' "économisation du monde " (Cardon et Cassili, 2015, p. 56). Traditionnellement, les luttes contre l'exploitation du capitalisme se sont plutôt articulées vers la réduction du temps de travail. " L'émancipation du travail salarié doit advenir 
Revendiquer le salariat ou comment court-circuiter le cycle d'accumulation du capital des médias sociaux numériques

grâce à la réduction du temps de travail sur la marge intensive » (Ackerman, 2016, p. 45).

Le problème, comme le démontre notre analyse, est que le capitalisme numérique s'est adapté à cette revendication. Le danger principal aujourd'hui, du moins pour les travailleurs occidentaux qui ont atteint un certain niveau de confort et qui n'ont pas à subir les affres de la société industrielle ou minière, n'est pas de trop travailler, mais bien de voir son temps de loisir transformé en un temps de travail. II ne sert à rien de réduire son temps de travail si son temps de loisir est consacré à faire du prosuming pour Google ou Facebook.

Considéré comme un moyen pour une lutte plus large, un nouveau salaire pourrait être très utile pour freiner le cycle d'accumulation du capital et donc enrayer le capitalisme. Nous allons ici nous inspirer des arguments du pamphlet Des salaires pour les étudiants publié en 1975. Ce pamphlet a été créé par des militants qui revendiquaient le salariat étudiant, eux-mêmes inspirés par la campagne pour le salariat des femmes ménagères dans les années 1970. En introduction d'une nouvelle édition, les auteurs écrivent :

Nous comprenions que même si la revendication d'un salaire étudiant, comme beaucoup de luttes pour l'augmentation des salaires, n'était pas en soi révolutionnaire, mettre fin au travail «non-salarié » [sic] sous toutes ses formes déstabiliserait, voire renverserait, le système capitaliste puisque ce secteur générait énormément de plus-value (Caffentzis et al., 2016, p. 149).

Pour ces militants, comme pour ceux qui militaient pour le salaire du travail ménager, être payés pour étudier représentait en fin de compte un plus grand pouvoir pour refuser le travail imposé par le capital. Comme l'affirme Federici (1975) dans le manifeste Wages against Housework, le but de revendiquer le salariat pour le travail ménager n'était pas de confiner les femmes à leur cuisine, mais bien de faire un premier pas vers le refus de ce travail. "Le salaire est nécessaire pour rendre le travail visible, puis pour s'organiser contre ce dernier » (Robert, 2014, p. 19).

Évidemment, les utilisateurs de médias sociaux sont une population très hétérogène qui regroupe sans aucun doute des 
individus très pauvres et très riches. Or dans une société où une grande partie des travailleurs se retrouvent dans un " précariat », c'est-à-dire dans une catégorie de citoyens qui accumulent les petits boulots à temps partiel et les contrats pour survivre (Durand Folco, 2017), un salaire supplémentaire pourrait aider grandement à augmenter l'autonomie des citoyens.

Dans le pamphlet, les militants identifient que le salariat étudiant affectera immédiatement trois facteurs. Premièrement, ils n'auront pas à travailler autant, ce qui leur procurera un plus grand pouvoir de négociation avec le capital. Deuxièmement, ils jouiront d'un niveau de vie plus élevé. Troisièmement, et pour finir, ils augmenteront le salaire moyen dans les secteurs touchés par la présence de travailleurs sous-payés (Caffentzis et al., 2016).

Ce qui est important de retenir ici, c'est que le salaire n'est pas l'enjeu de la lutte, ce n'est pas une fin. C'est plutôt un moyen de dénoncer une réalité d'exploitation. C'est une question d'éducation par rapport au travail. Parler de salaire, c'est ouvrir un champ nouveau pour comprendre les relations d'exploitation de manière différente : "Au lieu de considérer l'aliénation comme un vecteur de défaite, nous essayions, en transformant les études en relation salariale, d'un côté de reconnaître le travail scolaire comme une exploitation et de l'autre [de] faire reconnaître cette aliénation » (Caffentzis et al., 2016, p.202). Le but est de transformer l'aliénation en pouvoir.

C'est en ce sens que notre position est différente de celle des auteurs libertariens ou des hackers qui appellent également au salariat ou au droit commercial en échange des données (Lanier, 2013). Pour ces penseurs, la compensation, qui prendrait la forme de micro-redevances en échange d'un droit d'exploitation de nos données personnelles, est une fin en soi. Elle n'appelle pas à une prise de conscience plus large. II s'agit d'une vision libérale qui ne tente pas de stimuler l'agentivité des travailleurs.

Pour finir, des collègues marxistes pourraient, à la lecture de ce travail, critiquer notre démarche en affirmant que le fait de réclamer un salaire entre en contradiction avec la théorie de la valeur, qui suppose une remise en question des abstractions réelles que sont la valeur, le travail, la marchandise et le capital 
Revendiquer le salariat ou comment court-circuiter le cycle d'accumulation du capital des médias sociaux numériques

pour élaborer un après-capitalisme, mais, pour nous, le fait de réclamer compensation est justement l'un des meilleurs moyens d'ébranler les fondations du capitalisme. Comme l'affirme le théoricien marxien Anselm Jappe (2009), « la valeur n'existe, et ne peut exister, que dans un rapport dialectique avec la non-valeur, et ce rapport est nécessairement antagoniste » (p. 106).

La valeur ne peut progresser que par l'exploitation de la nonvaleur. Le travail non salarié est aujourd'hui l'un des plus grands vecteurs de plus-value. Beaucoup de stratégies d'accumulation différentielle (comme la publicité, l'image de marque, la recherche et le développement, l'innovation, l'intermédiation financière) repose aujourd'hui sur des mécanismes qui peuvent très bien être encouragés par le travail non salarié, par exemple celui des développeurs de logiciels libres (Broca, 2013 ; Nitzan et Bichler, 2012) Si ce secteur disparaît, c'est tout le capitalisme qui se verrait fortement ébranlé. Cette stratégie est pour nous la plus utile et la plus efficace pour élaborer cet après-capitalisme désiré par tant de théoriciens marxiens.

\section{Conclusion}

Notre travail détaille un moyen d'enrayer le cycle d'accumulation du capital des grands médias sociaux du capitalisme numérique. Cet arrêt, ou ce ralentissement du cycle d'accumulation, n'est pas seulement urgent pour mettre fin à l'exploitation individuelle des prosumers, il est aussi important pour la société et la démocratie en général. Le processus d'accumulation du capital de ces entreprises est une opération de surveillance économique (Andrejevic, 2013). Nous avons laissé des "machines ultimes de surveillance économique " (Fuchs, 2012, p. 45) se développer. En plus de nous surveiller, ces multinationales ont considérablement affaibli nos systèmes de représentation traditionnels. Loin d'encourager l'autonomie collective et l'auto-institution de la société, le modèle des GAFAM tend à nous enfermer dans des réseaux privés où la liberté humaine est réduite à un comportement « adaptatif de type réactif ou béhavioriste " (Freitag, 2011, p. 16) face aux codes, aux prix ou aux spectacles qui nous sont présentés de manière permanente. 
La question est aussi de savoir ce que fait Google avec 32 milliards de revenus. Loin de redistribuer cette richesse, les propriétaires de cette compagnie tentent de réaliser leur fantasme en investissant des sommes colossales dans des projets relevant du transhumanisme (Alexandre, 2013). Nous croyons que le meilleur moyen de "faire trembler » Google ou Facebook n'est pas de lancer une campagne de boycottage ni de construire des solutions de rechange locales qui tenteraient de faire sécession, mais bien de pointer du doigt la réalité d'exploitation de ces compagnies et de demander compensation. Le seul moyen de faire fléchir ces compagnies est de court-circuiter leur cycle d'accumulation du capital.

Comme l'affirme Christian Fuchs (2012, 2020), les possibilités des GAFAM pointent au-delà du capitalisme. Ils sont le meilleur et le pire de ce qui est arrivé à Internet. Le problème n'est pas les services de ces entreprises, mais bien les rapports capitalistes de production. II est nécessaire de réactiver des luttes et des revendications pour détruire le pied d'argile de ces géants. 
Revendiquer le salariat ou comment court-circuiter le cycle d'accumulation du capital des médias sociaux numériques

\section{Références}

Ackerman, S. (2016). L'allocation universelle comme solution au chômage?. Dans M. Alaluf et D. Zamora (dir.), Contre l'allocation universelle (p. 37-46). Montréal, Canada : Lux.

Alexandre, L. (2013, 18 avril). Google et les transhumanistes. Le Monde. Repéré à https://www.lemonde.fr/sciences/article/2013/04/18/googleet-les-transhumanistes 3162104 1650684.html

Andrejevic M. (2013). Estranged Free Labor. Dans T. Scholz (dir.), Digital Labor: The Internet as Playground and Factory (p. 149-164). New York, NY : Routledge.

Arvidsson, A. et Colleoni, E. (2012). Value in Informational Capitalism and on the Internet. The Information Society, 28(3), 135-150.

Bekhtaoui, A. (2019, 25 avril). Microsoft franchit les 1000 milliards en Bourse grâce à l'infonuagique. La Presse. Repéré à https://www.lapresse.ca/affaires/entreprises/201904-25/microsoft-franchit-les-1000-milliards-en-bourse-gracea-l-infonuagique

Belleflamme, P. et Toulemonde, E. (2018). Tax incidence on competing two-sided platforms. Journal of Public Economic Theory, 20(1), 9-21.

Bolin, G. (2010). Symbolic production and value in media industries. Journal of Cultural Economy, 2(3), 345-361.

Braverman, H. (1976). Travail et capitalisme monopoliste. Paris, France : Maspero.

Broca, S. (2013). Utopie du logiciel libre. Du bricolage informatique à la réinvention sociale. Paris, France: Le Passager Clandestin.

Broca, S. (2018, janvier). Les communs comme critique du capitalisme numérique. Des ressources partagées à un nouveau mode de production?. Communication présentée au Séminaire de l'axe 4, Épistémologie critiques en culture et en communication, du CRICIS, Montréal, Québec.

Caffentzis, G. et al. (2016). Des salaires pour les étudiants. Toulouse, France : Éditions de l'Asymétrie. 
Cardon, D. et Casilli, A. A. (2015). Qu'est-ce que le digital labor?. Bry-sur-Marne, France : INA.

Casilli, A. A. (2019). En attendant les robots: enquête sur le travail du clic. Paris, France : Éditions du Seuil.

Castells, M. (2001). La société en réseaux: l'ère de l'information (nouvelle édition). Paris, France : Fayard.

Durand Folco, J. (2017). À nous la ville! Traité de municipalisme. Montréal, Canada : Les Éditions Écosociété.

De Kosnik, A. (2013). Fandom as Free Labor. Dans T. Scholz (dir.), Digital Labor: The Internet as Playground and Factory (p. 98-111). New York, NY : Routledge.

Dujarier, M-A. (2008). Le travail du consommateur. De McDo à eBay: comment nous coproduisons ce que nous achetons. Paris, France : La Découverte.

Durand, C. (2018). L'envers de l'économie numérique: un capitalisme intellectuel monopoliste. Note de recherche de I'IFRIS, (7).

Federici, S. (1975). Wages against housework. Bristol, Royaume-Uni : Falling Wall Press.

Freitag, M. (2011). L'abîme de la liberté. Critique du libéralisme. Montréal, Canada : Liber.

Fuchs, C. (2010). Labor in Informational Capitalism and on the Internet. The Information Society, 26(3), 179-196.

Fuchs, C. (2011). Web 2.0, prosumption, and surveillance. Surveillance \& Society, 8(3), 288-309.

Fuchs, C. (2012). Google capitalism. tripleC: Communication, Capitalism \& Critique. Open Access. Journal for a Global Sustainable Information Society, 10(1), 42-48.

Fuchs, C. (2020). Towards a critical theory of communication as renewal and update of Marxist humanism in the age of digital capitalism. Journal for the Theory of Social Behaviour. doi: $10.1111 /$ jtsb. 12247

Goyette-Côté, M.-O. (2013). Les nouvelles formes du travail, ou comment la notion de "prosumer » permet d'analyser les pratiques participatives sur I'Internet. Dans O. Kane, É. George et N. Naoufal (dir.), Où [en] est la critique en 
Revendiquer le salariat ou comment court-circuiter le cycle d'accumulation du capital des médias sociaux numériques

communication? Actes du colloque international du $80^{\text {ème }}$ congrès de l'Association francophone pour le savoir (Acfas), Palais des congrès de Montréal, 7 au 11 mai 2012 (p. 150-162). Montréal, Canada : GRICIS UQAM.

Goyette-Côté, M-A. et Langlois, P. (2014). La participation sur les espaces de contenus générés par les utilisateurs, une exploitation collaborative, tic\&société, 8(1-2). Repéré à http://journals.openedition.org/ticetsociete/1407

Hesmondhalgh, D. (2010). User-generated content, free labour and the cultural industries. ephemera, 10 (3-4), 267-284.

Horkheimer, M. et Adorno, T. W.. (1983). La dialectique de la raison fragments philosophiques. Paris, France : Gallimard.

Howe, J. (2006). The Rise of Crowdsourcing. Wired, 14(06). Repéré à http://www.wired.com/wired/archive/14.06/crowds pr.html

Huws, U. (2014). Labor in the global digital economy: The cybertariat comes of age. New York, NY: New York University Press.

Jappe, A. (2009). Le "côté obscur " de la valeur et le don. Revue du MAUSS, 34(2), 96-113.

Jappe, A. (2011). Crédit à mort: la décomposition du capitalisme et ses critiques. Paris, France : Lignes.

Jarrett, K. (2015). Feminism, labour and digital media: The digital housewife. New York, NY : Routledge.

Lanier, J. (2013). Who owns the future?. New York, NY : Simon \& Schuster.

Lazzarato, M. (2011). La fabrique de l'homme endetté : essai sur la condition néolibérale. Paris, France : Amsterdam.

Lomazzi, L. et Menard, M. (2018). Où en est la théorie du/des commun(s)? Vers une économie politique culturelle. tic\&société, 12(1), 69-93.

Manzerolle, V. et McGuigan, L. (dir.). (2014). The audience commodity in a digital age: Revisiting a critical theory of commercial media. New York, NY : Peter Lang. 
Martin, É. et Ouellet, M. (2014). La tyrannie de la valeur: débats pour le renouvellement de la théorie critique. Montréal, Canada : Les Éditions Écosociété.

Marx, K. (1977). Le Capital. Livre premier. Paris, France: Éditions sociales.

Mosco, V. (2008). Knowledge Workers of the World! Unite?. Canadian Journal of Communications, 33, 121-125.

Nitzan, J. et Bichler, S. (2012). Le capital comme pouvoir. Paris, France : Max Milo Éditions.

Nixon, B. (2014). Toward a political economy of "audience labour" in the digital era. tripleC: Communication, Capitalism \& Critique. Open Access Journal for a Global Sustainable Information Society, 12(2), 713-734.

Ouellet, M. (2016). La révolution culturelle du capital. Montréal, Canada : Les éditions Écosociété.

Ouellet, M. (2015). Revisiting Marx's Value Theory: Elements of a Critical Theory of Immaterial Labor in Informational Capitalism. The Information Society, 31(1), 20-27.

Ouellet, M., Ménard, M., Bonenfant, M. et Mondoux, A. (2015). Big Data et quantification de soi : la gouvernementalité algorithmique dans le monde numériquement administré. Canadian Journal of Communication, 40(4), 597-613.

Piccone, P. (1975). Reading the Grundrisse: Beyond "orthodox" Marxism. Renewal and Critique in Social Theory, 2(1), 235255.

Pineault, É. (2008). Quelle théorie critique des structures sociales du capitalisme avancé ?. Cahiers de recherche sociologique, (45), 113-132.

Ritzer, G. (1983). The "McDonaldization" of Society. Journal of American Culture, (6), 100-107.

Robert, C. (2014). Le salaire au travail ménager: réflexion critique sur une lutte oubliée. Les féminismes d'hier à aujourd'hui. POSSIBLES, 38(1), 13-26.

Saleh, I. (2017). Les enjeux et les défis de l'Internet des Objets (IdO). Internet des objets. Repéré à https://www.openscience.fr/IMG/pdf/iste idov1n1 1.pdf 
Revendiquer le salariat ou comment court-circuiter le cycle d'accumulation du capital des médias sociaux numériques

Salvet, J.-M. (2018,13 mars). Projet de loi sur un « revenu de base ». Le Soleil. Repéré à https://www.lesoleil.com/ actualite/politique/projet-de-loi-sur-un-revenu-de-base824cd07734eeccd516f1168f9947a0f0

Sandoval, M. (2020). Entrepreneurial activism? Platform cooperativism between subversion and co-optation. Critical Sociology, 46(6), 801-817.

Scholz, T. (dir.). (2013). Digital Labor. The Internet as Playground and Factory. New York, NY : Routledge

Scholz, T. (2017). Uberworked and Underpaid. Cambridge, Royaume-Uni : Polity.

Smyrnaios, N. (2017). Les GAFAM contre l'Internet: une économie politique du numérique. Paris, France : INA.

Smyrnaios, N. et Rebillard, F. (2019). How infomediation platforms took over the news: A longitudinal perspective. The Political Economy of Communication, 7(1), 30-50.

Smythe, D. W. (1977). Communications: Blindspot of western Marxism. CTheory, 1(3), 1-27.

Srnicek, N. (2018). Capitalisme de plateforme. L'hégémonie de l'économie numérique. Montréal, Canada : Lux.

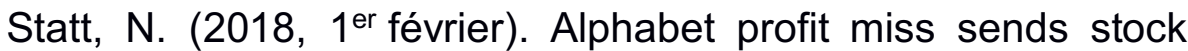
down, but Google ad and cloud sales remain strong. The Verge. Repéré à https://www.theverge.com/2018/2/1 /16961214/alphabet-google-q4-2017-earnings-reportannouncement-youtube

Stiegler, B. et Kyrou, A. (2016). Le revenu contributif et le revenu universel. Multitudes, (63), 51-58.

Terranova, T. (2000). Free labor: Producing culture for the digital economy. Social Text, 18(2), 33-58.

Toffler, A. (1980). La Troisième vague. Paris, France : Denoël.

Vioulac, J. (2018, 12 mars). Contre la machine capitaliste. Grozeille. Repéré à https://grozeille.co/vioulac/

Wood, E. M. (2002). The origin of capitalism: A longer view. New York, NY : Verso. 


\section{Samuel LAMOUREUX}

Zamora, D. (2016). Histoire et genèse d'une idée néolibérale. Dans M. Alaluf et D. Zamora (dir.), Contre l'allocation universelle (p. 13-36). Montréal, Canada, Lux. 